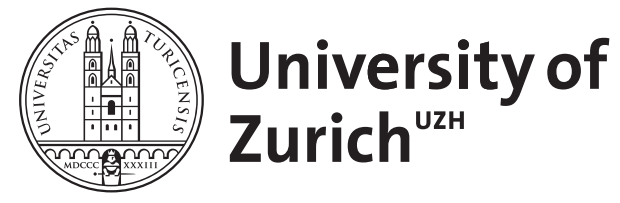

Zurich Open Repository and Archive

University of Zurich

University Library

Strickhofstrasse 39

CH-8057 Zurich

www.zora.uzh.ch

Year: 2017

\title{
Medea
}

Berndt, Frauke

DOI: https://doi.org/10.1515/9783110332681-012

Posted at the Zurich Open Repository and Archive, University of Zurich

ZORA URL: https://doi.org/10.5167/uzh-162020

Book Section

Originally published at:

Berndt, Frauke (2017). Medea. In: Berndt, Frauke; Goebel, Eckart. Handbuch Literatur Psychoanalyse. Berlin, Boston: De Gruyter, 218-240.

DOI: https://doi.org/10.1515/9783110332681-012 


\section{III.3. Medea}

\section{Frauke Berndt}

\section{Einleitung: Euripides' Medeia}

Seit zweieinhalb Jahrtausenden macht die Kindermörderin mehr als jede andere mythologische Figur in Literatur und anderen Medien Karriere (vgl. u. a. Lütkehaus 2007; Luserke-Jaqui 2002; Stephan 2006). Dramen, Romane, Erzählungen, Hörspiele, Bilder und heute vor allem Filme bewahren die Pathosformel im kulturellen Gedächtnis. Medea liebt, raubt das Goldene Vlies und flieht mit ihrem Ehemann Jason aus Kolchis westwärts. Auf dem Weg beginnt sie zu morden: erstens ihren Bruder Absyrtos, zweitens Jasons Onkel Pelias in Thessalien, drittens seine neue Braut Kreusa, viertens deren Vater Kreon, den König von Korinth, sowie schließlich fünftens und sechstens ihre beiden mit Jason gezeugten Söhne. Dieses Narrativ geht auf Euripides zurück, dessen Medeia es 431 v. Chr. im Wettkampf der Tragödiendichter freilich nur auf den dritten Platz geschafft hat: „Er entrückt Medea dem Mythos und stellt sie in einen für den Zuschauer realen Wirklichkeitsbereich.“ (Kenkel 1979, 22; vgl. Schmierer 2005)

Die Transformation von Mythos in Tragödie basiert auf der Festlegung von drei zentralen Figuren, Medea, Jason und Kreon, der straff durchgearbeiteten Handlung, insbesondere aber der Fokussierung auf den ,ganz spezifischen tragischen Kern“ (Böschenstein 1995, 129; $\rightarrow$ IV.1. TRAGöDIE) der Morde an den beiden Söhnen. Dabei scheidet die Rezeptionsgeschichte Freunde von Feindinnen, die dem „Lohnschreiber“ Euripides vorwerfen, dass er Medea den „Kindermord unterjubelt“: „[S]eitdem jagen sie dich durch unsere Literaturen“ (Novak 1983 [1977], 96), empört sich Helga Novak 1977 im Brief an Medea. „Die Möglichkeit, daß sich die Mutterliebe, Garantin der Sicherheit des Kindes, in todbringende Aggression verkehren kann, ist zumindest in der europäischen Kultur schwerer artikulierbar als Vater- und Muttermord“ (Böschenstein 1994, 8), kommentiert Renate Böschenstein das in der griechischen Mythologie eher seltene Verbrechen. Trotzdem mordet Medea bei Euripides weder als (biologische) Frau noch als Mutter noch als Wahnsinnige noch als Fremde. Solche modernen Motivationen des tragischen Fehlers einer Figur (hamartia) entwickeln sich erst im Lauf der Zeit. Bei Euripides hat Medea drei gute Gründe für die Kindermorde: Weil Jason sie verlassen hat, um Kreusa zu heiraten, mordet Medea - legitimiert durch den Chor der korinthischen Frauen - als Ehefrau, als Königstochter und nicht zuletzt als Enkelin des Sonnengottes. Durch die um Kreusa erweiterten Kindermorde löscht sie die Dynastie der Jasoniden aus, was im antiken Horizont ein

https://doi.org/10.1515/9783110332681-012

Bereitgestellt von | provisional account

Angemeldet

Heruntergeladen am | 01.02.19 11:18 
ethisch, politisch und psychologisch ebenso nachvollziehbares wie vor allem äußerst wirksames Mittel der Rache darstellt (vgl. Gabriel 1992):

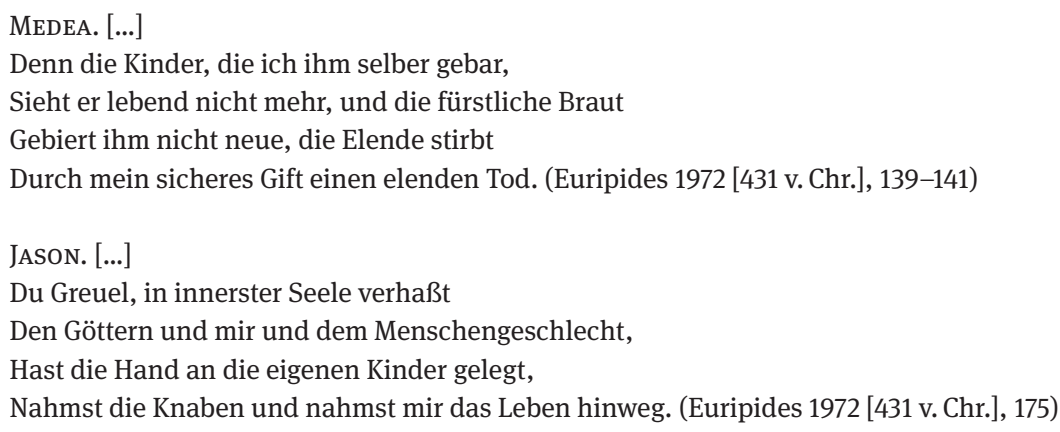

Inge Stephan fasst vier aktuelle Interpretationsmodelle des Stoffes zusammen: Heute dient Medea als Identifikationsfigur des Geschlechterkampfes, als Bewältigungsfigur krisenhafter geopolitischer und/oder familialer Ordnungen, als Projektionsfigur der Alterität sowie als Reflexionsfigur ethischer Probleme, vor allem der Legitimität von Gewalt (vgl. Stephan 2006, 5). Verschiebung, Ausblendung, Entsühnung (Buße), Umdeutung, Psychologisierung, Pathologisierung und Heroisierung (Vergöttlichung) der Kindermorde sind die literarischen Strategien, die von der Antike bis in die Gegenwart das heterogene Feld der Medea-Bearbeitungen prägen (vgl. Stephan 2006, 2-3). Dabei zeichnen sich für den Zusammenhang von Literatur und Psychoanalyse folgende Eckdaten ab: Im 18. Jahrhundert errichtet die Literatur das bürgerliche Tabu des Kindsmordes (vgl. Abschnitt 2.). Dieses Tabu übersetzt die Psychoanalyse des 20. Jahrhunderts in die Diagnose des sogenannten Medeakomplexes (vgl. Abschnitt 3.). Im kulturellen Imaginären hängt dieser Komplex wiederum von einer chthonisch-maternalen Imago ab, die Euripides mit seiner Tragödie verdeckt (vgl. Abschnitt 4.). Individuell Unbewusstes und kulturell Unvordenkliches fallen schließlich in einer Leerstelle zusammen, die sowohl Film als auch Literatur, nach Freud' zu füllen versuchen (vgl. Abschnitt 5.).

\section{Kindermorde vs. Kindsmord}

Mit den Kindermorden werfen die Medea-Bearbeitungen seit alters her sowohl ethische als auch ästhetische Probleme auf. Bereits in der Ars poetica (14 v. Chr.) mahnt Horaz Sensibilität für die Leistung verschiedener Medien gegenüber bestimmten Stoffen an: 
Trotzdem laß Dinge, die ins Haus gehören, nicht vor der Bühnenwand geschehen; laß vieles den Augen entrückt bleiben: dann mag beredter Zeugenmund es anschaulich erzählen. Nicht darf vor allem Volk Medea ihre Kinder schlachten; nicht darf der grausige Atreus Menschenfleisch auf offner Bühne kochen, nicht Prokne in den Vogel, Kadmus in die Schlange sich verwandeln. (Horaz 1967 [14 v. Chr.], 241)

Die antike Darstellungsscheu ist also nicht per se ein „Syndrom“, „das sich am besten im Rückgriff auf das kulturanthropologische Konzept des ,Tabus‘ erläutern lässt“ (Port 2008, 103), wie Ulrich Port nahelegt, sondern liegt in einer ästhetischen Norm begründet. Das juristische Kindsmord-Delikt ist eine neuzeitliche Erfindung (vgl. Mauerer 2002, 151-204). In diesem Sinn behandelt auch Euripides die Tötung von zwei älteren Kindern als Verbrechen, zumal die Mörderin als Frau im heutigen Sinn weder rechts- noch geschäftsfähig ist, wie Medea vor dem Chor der korinthischen Frauen klagt (vgl. Euripides 1972 [431 v. Chr.], 167-169). Wenn daher Euripides' Medea ihre beiden Söhne nicht auf offener Szene töten lässt, dann hat diese Beschränkung in erster Linie ästhetische Gründe, die nicht einfach mit ethischen verwechselt werden dürfen. Weil Medea nicht strafmündig ist, wird aus dem rechtlichen Fall ein psychologischer. Die Motivation der Kindermorde steht daher seit Seneca im Zentrum des Interesses an Medea. Auf der Grundlage der drei Bücher seines Werkes De ira (1.Jh. n. Chr.) behandelt er die (biologische) Frau als Beispiel mangelnder Affektkontrolle. Medeas sexuelles Begehren nach Jason und ihre Rache an ihm bilden die Kehrseiten der psychologischen Medaille, die seitdem gängige Währung in der Motivation der Kindermorde ist. Zum Schutz der Kinder müssen die bedrohliche weibliche Leidenschaft gezügelt und die Frauen selbst unter männliche,Fürsorge‘ gestellt werden. Dabei leistet die Mythologie Seneca noch ein wenig Schützenhilfe, indem er auf die Furien zurückgreift, die den Schatten des Absyrtos, das heißt den toten Bruder begleiten. Medea sühnt den Brudermord, der gegen das mythologische Blutrecht verstößt, mit dem Mord ihres ersten Sohnes, um erst den zweiten im psychologischen Konflikt mit Jason abzuschlachten. Erst Pierre Corneille setzt Senecas Psychologisierung vollends um. Dabei erweitert er einerseits den Eintrag ,Medea in der Enzyklopädie um mythologisches Wissen, das die Quellen bis zum 17. Jahrhundert erschlossen haben. Andererseits tritt nun erstmals Kreusa auf die Bühne, sodass die Beziehung zwischen Medea und Jason trianguliert wird. Das doppelte mimetische Begehren verbindet die triumphierende Kreusa mit der eifersüchtigen Medea und kompliziert die psychologische Motivation der Kindermorde.

Die von Horaz geforderte mediale Selbstbeschränkung gilt bis in das 18. Jahrhundert, nun allerdings unter veränderten Rahmenbedingungen. In seinem Laokoon oder über die Grenzen der Mahlerey und Poesie (1766) beobachtet Gotthold Ephraim Lessing, wie der Maler Timomachus das Darstellungsproblem löst: 
„Die Medea hatte er nicht in dem Augenblicke genommen, in welchem sie ihre Kinder wirklich ermordet; sondern einige Augenblicke zuvor, da die mütterliche Liebe noch mit der Eifersucht kämpfet.“ (Lessing 1990 [1766], 33) Dementsprechend stellen auch die berühmten modernen Gemälde von Eugène Delacroix (1864), Henri Klagman (1868) oder Anselm Feuerbach (1870; 1873) Medea stets vor den Morden an den beiden Kindern dar. Weil Medea im 18. Jahrhundert ohne jede Einschränkung strafmündig ist, wird aus dem psychologischen ein forensischer Fall, wie vor allem diejenigen Medea-Bearbeitungen zeigen, in denen das Verbrechen aus dem Handlungszusammenhang des Medea-Stoffes gelöst wird: Lessings Miß Sara Sampson (1755), Heinrich Leopold Wagners Die Kindsmörderin (1766) sowie Johann Wolfgang Goethes Faust I in der Gretchen-Tragödie (1808).

Mit Friedrich Maximilian Klinger zieht Medea aus dem Gefängnis endgültig in die Klinik um - die Diagnose lautet Wahnsinn (heute: Schizophrenie): „Ich benuzte weder die griechische, noch die lateinische, noch die französische Medea. Diese hier, und wie sie sey, ist mein Werk“ (Klinger 2012 [1786], 5), annonciert Klinger die erste seiner beiden Tragödien Medea in Korinth (1786), in der er sich nichtsdestotrotz großzügig in der Literaturgeschichte bedient, insbesondere aber Senecas und Corneilles psychomythologische Tricks verfeinert. Medea wird von ,Hecates Stimme‘, die außer ihr keine andere dramatische Figur hört, die also nur ,in ihrem Kopf‘ wütet, zur Rache aufgefordert. Der dreifachen Sühne dienen die Kindermorde: erstens des Mordes an Hekates Sohn, Medeas Bruder Absyrtos, zweitens des Mordes an einem anderen Säugling und erst drittens - in Bezug auf Medea - der Sühne von Jasons Verrat:

\footnotetext{
Hecates Stimme: Nah' dich den Schläfern! [...] Durch sie räche dich! Lege des Erebos Flammen durch ihren Tod an des Meineidigen Herz. Zehre auf sein Gehirn! [...]

MEDEA: Ha, Mutter, es ist auch mein Blut! [...]

Hecates Stimme: Medea! vollziehe die Rache! [...]

MEDEA: Ich bin Medea, bin nicht eure Mutter. Seht dort, die Nacht-Eule auf der Cypressen Spitze! sie will euch haben! Lechzt nach eurem Blut, und zieht euch in ihrer düstren Wolke in Erebos. (Klinger 2012 [1786], 61-65)
}

Mit Medeas „furchtbare[m] Selbst“ (Klinger 2012 [1786], 88) geht gleichzeitig im besten Sinn eine Entschuld(ig)ung einher: Die Eumeniden (Furien) jagen nicht Medea, sondern Kreusa und Jason durch den fünften Akt. Erst mit Medeas Umzug in die Klinik greift das Phänomen, das man in Freuds Sinn tatsächlich als Tabu bezeichnen kann, weil der Kindsmord auf dem Weg der Psychologisierung in der Epoche der Aufklärung zu etwas geworden ist, „was zugleich heilig, über das Gewöhnliche erhaben, wie auch gefährlich, unrein, unheimlich“ (IX, 31) ist. In vier Aufsätzen unter dem Titel Totem und Tabu. Einige Übereinstimmungen im Seelenleben der Wilden und der Neurotiker (1913) beschreibt Freud, wie Tabus in 
Bezug auf „Handlungen, Artikulationsweisen oder Verhaltensformen“ errichtet werden, ,die der Sitte nach untersagt und nicht selten auch per Gesetz verboten sind“ (Benthien und Gutjahr 2008, 7; $\rightarrow$ II.5. KulturTHEORIE). Auch die bürgerliche Wertegemeinschaft schützt sich mit einem solchen Meidungsgebot - und zwar vor ihrer eigenen ideologischen Überforderung universaler Humanität. Sie stellt sich im 18. Jahrhundert einerseits mit dem ethischen Konzept der ,romantischen' Liebe (vgl. Luhmann 1982), andererseits mit demjenigen der ,natürlichen' Mutterliebe ein (vgl. Badinter 1980). Als Ergebnis des einen und Gegenstand des anderen wird ,das Kind' in der bürgerlichen Familie zum prekären Objekt. Doch weder romantische Liebe noch Mutterliebe sind universal. Wenn wir Medea heute an der Elle der Humanität messen, dann ebnen wir alle historischen sowie kulturellen Unterschiede in der Motivation der Kindermorde einfach ein. Die Figur Medea hat indes, bei allem was sie tut oder lässt, immer einen spezifischen soziokulturellen Gender-Index (vgl. Benthien und Gutjahr 2008, 11; $\rightarrow$ II.7. GENDER UND QUEER STUDIES). Die moderne Affektökonomie, in deren Zentrum Schuld und Scham der Protagonistin stehen, geht daher einzig und allein auf das Konto eines bürgerlichen Tabus, keines universalen.

In der „hohe[n] Zeit der Mutterliebe“ (Lütkehaus 2007, 16) spannt auch Franz Grillparzer in seiner Trilogie Das goldene Vließ (1822) den Stoff zwischen zwei Achsen auf: Die horizontale inszeniert - im Zeichen des Historismus - den kulturellen Konflikt zwischen Kolchis und Korinth, Matriarchat und Patriarchat, ,Barbarentum' und Zivilisation, die vertikale den ethischen Konflikt zwischen Humanität und Inhumanität ( $\rightarrow$ II.6. Postcolonial UNd CRITICAL RACE STUdies). In der Figur Medea überschneiden sich die beiden Achsen, weil die Kolcherin humaner ist als die Korinther, die sie als Fremde ausschließen. Selten finden sich die Kindermorde so schlecht motiviert wie bei Grillparzers ,verteufelt humaner Heldin, sodass er einen dramaturgischen Trick bemühen muss. Medeas zunächst vergrabene Symbole magischer Potenz gelangen im vierten Akt - wieder ausgegraben - ,zufällig' zu ihr zurück. Nachdem Kreusa nicht nur die empfindsamere Freundin, sondern auch die bessere Mutter zu sein verspricht, ermordet die verbannte Medea ihre Kinder, um sie der Rache der Korinther zu entziehen. Gleichzeitig verschiebt Grillparzer wie Klinger die moralische Schuld von Medea auf Jason. Als im 19. Jahrhundert nun christliche Büßerin will sich Medea-Magdalena in Delphi dem Gott Apollon stellen, der im deutschsprachigen Klassizismus die Humanitätsidee verbürgt, und fordert auch Jason auf, sich zu seiner Schuld zu bekennen: „Trage! [...] Dulde! [...] Büße! Ich geh’ und niemals sieht dein Aug mich wieder!““ (Grillparzer 1986 [1822], 390)

Am Ende dieses Prozesses universal (miss-)verstandener Humanisierung steht Christa Wolf, die in Medea. Stimmen (1996) reinen Tisch macht: Medea ist keine Zauberin mehr, sondern Heilkundige, keine eifersüchtige Frau, sondern 
sexuell befriedigt in neuer Partnerschaft, keine Wahnsinnige, sondern Psychotherapeutin. Im polyphonen Arrangement wird sie von den Kindermorden freigesprochen, die Wolf im Dialog mit René Girards Le Bouc émissaire (1982; Der Sündenbock) als Ergebnis einer politischen Intrige der patriarchalen Monarchie gegen die matriarchale Tradition darstellt. Die Rädelsführer machen sich die unaufgeklärte Fremdenfeindlichkeit des Volkes zunutze. Während Medea dem Pöbel nur mit knapper Not entkommt, ermorden die Korinther die Kinder, weil sie die Fremden als Sündenböcke für die Pest in der Stadt verantwortlich machen. Menschlicher kann Medea nicht mehr werden!

\section{Medeakomplex - Medea-Phantasie - Medea-Konfiguration}

In der Traumdeutung (1900) bezeichnet Sigmund Freud die Kindermorde als „spezifisch weibliche[s] Verbrechen“ (II/III, 162). Dennoch interessieren ihn weder das bürgerliche Tabu des Kindsmordes noch Euripides' Medea als paradigmatische Figur. Bekanntermaßen ist sein Modell psychischer Entwicklung auf das männliche Kind zugeschnitten. Im Hinblick auf die Triebökonomie des weiblichen Kindes entscheidet er sich für Elektra ( $\rightarrow$ III.4. ELEKTRA). Doch obwohl er sich für Medea so auffallend nicht interessiert, assoziiert er sie 1905 im Bruchstück einer Hysterie-Analyse (vgl. V, 222) eher beiläufig in einer seiner Fallgeschichten $(\rightarrow$ IV.6. FALLGESCHICHTE). Ida Bauer (Pseudonym: Dora), die an den Symptomen einer ausgeprägten Hysterie litt, wurde von ihrem Vater Philipp Bauer mit dessen verheiratetem Freund Hans Zellenka (Pseudonym: Herr K.) verkuppelt, damit er selbst seine Affäre mit Zellenkas Frau Peppina (Pseudonym: Frau K.) ungestört ausleben konnte. Man habe sie geopfert, so empfindet die Patientin. An einer Stelle im Bericht assoziiert Freud Frau K. und Dora mit Medea und Kreusa - an derjenigen Stelle, an der Frau K. nämlich Dora die beiden Kinder quasi überlässt:

\footnotetext{
Ich erfuhr dann, daß die junge Frau und das kaum erwachsene Mädchen Jahre hindurch in der größten Vertraulichkeit gelebt hatten. Wenn Dora bei den K. wohnte, teilte sie das Schlafzimmer mit der Frau; der Mann wurde ausquartiert. Sie war die Vertraute und Beraterin der Frau in allen Schwierigkeiten ihres ehelichen Lebens gewesen; es gab nichts, worüber sie nicht gesprochen hatten. Medea war ganz zufrieden damit, daß Kreusa die beiden Kinder an sich zog; sie tat gewiß auch nichts dazu, um den Verkehr des Vaters dieser Kinder mit dem Mädchen zu stören. (V, 222; Hervorh. F. B.)
}

Die Konfiguration ist insofern bizarr, als Kreon (Vater), Medea (Frau K.) und Kreusa (Dora) ein familiales Dreieck bilden. Als Medea rückt Frau K. dabei an die Stelle von Doras Mutter, die an einer „,Hausfrauenpsychose““ litt (V, 178), 
sodass die Tochter keine stabile Bindung zu ihr aufbauen konnte, während sie eine innige Bindung zum Vater hatte. Zwei Träume erzählt Freud nach. Besonders der erste verdichtet Medea-Material, ohne dass Freud indes darauf aufmerksam wird, weil er das heuristische Potenzial des Medea-Stoffes - wie gesagt - nicht bemerkt:

In einem Haus brennt es [...], der Vater steht vor meinem Bett und weckt mich auf. Ich kleide mich schnell an. Die Mama will noch ihr Schmuckkästchen retten, der Papa sagt aber: Ich will nicht, daß ich und meine beiden Kinder wegen deines Schmuckkästchens verbrennen. Wir eilen herunter, und sowie ich draußen bin, wache ich auf. (V, 225)

Hinter dem Traum stehe der Konflikt der eifersüchtigen Dora, die der Frau von K., die Gegenstand ihres homosexuellen Interesses sei, den Vater nicht gönne, diesen gleichzeitig in Herrn K. begehre. Durch Verschiebungen und Verdichtungen inszeniere der Traum eine Wunscherfüllung: den Sexualakt mit Herrn K., dessen Verdrängung die Ursache der hysterischen Symptome sei. Freud entgeht freilich bei allen Tagesresten die Medea-Kulisse der Szene: das brennende Haus, in dem Kreusa stirbt, und das Schmuckkästchen, das nicht nur ein Symbol des weiblichen Genitals ist, sondern vor allem auch an die „Kiste“ (Grillparzer 1986 [1822], 307) erinnert, die Medeas magische Requisiten birgt. Tatsächlich sterben sowohl Kreusa als auch die Kinder am Inhalt dieser Kiste, das heißt (bleiben wir in der etwas kruden Sexualsymbolik) an Medeas Sexualität. Wenn der Traum also tatsächlich eine Wunscherfüllung darstellt, dann identifiziert sich Dora hier nicht mit Kreusa, sondern mit Medea, und dann drückt die Sorge um die ,beiden Kinder` Doras Angst vor ihrer eigenen Weiblichkeit aus, die Sexualität, Generativität und Destruktivität verbindet.

Wenn 1948 der Medea-Complex benannt wird, dann spielt Freuds Fallgeschichte in der Ätiologie keine Rolle (vgl. Roos 2015), obwohl der Traum gewissermaßen wie ein heimliches Emblem alles vorwegnimmt, was in den kommenden Jahrzehnten diskutiert wird. Sieben klinische Fälle kontextualisiert Edward S. Stern 1948 sowohl kulturanthropologisch als auch literarhistorisch, um in der Literaturgeschichte die Antwort auf die Frage zu finden: Warum morden Mütter? Die ambivalente Einstellung der Mutter gegenüber ihren Kindern setzt Stern in Beziehung zu ihrem Hass auf den (Ehe-)Mann.

(1) The situation in which the mother harbours death wishes to her offspring, usually as a revenge against the father, is described and named the Medea complex.

(2) It is shown that there is considerable resistance against admitting these thoughts to the consciousness of the mother or any other person, but that they are of general occurrence. 
(3) The Medea complex causes many marital difficulties, e.g., dyspareunia, prevention and interruption of pregnancy, failure of breast feeding, and other disordered domestic relations.

(4) It explains such matters as baby farming, disposal to others, and neglect of children, unjust accusations of cruelty to children such as blood libels, and acts of covert and overt cruelty to them. (Stern 1948, 330)

„Symbolically“, so bringt Robert Tyminski den Medeakomplex auf den Punkt, „aggression directed at children implies that growth is cut off, the next generation is eliminated, and prospects for the future are diminished“ (Tyminski 2014, 38). Auf klinischer Grundlage der postpartalen Depression (,Wochenbettdepression'), sogar Psychose geht der Komplex in die psychologische Typologie ein (vgl. u.a. Schaule 1982; Wiese 1993). Psychoanalytische Interpretationen im engeren Sinn setzen erst in den 1980er Jahren ein. Sie kreisen um das bürgerliche Tabu des Kindsmordes, dessen psychische Ökonomie sie ergründen. Wie die Analyse des Ödipuskomplexes beschränkt sich auch diejenige des Medeakomplexes auf einen einzigen Text: Euripides' Tragödie, mit der die Psychoanalyse als geschichtsblinde Disziplin eine Meta-Medea voraussetzt.

Einig ist man sich darüber, dass der Medeakomplex aus frühkindlichen Erfahrungen der mordenden Mutter resultiert. Simone Bécache versteht ihn 1982 als weiblichen Ödipuskomplex beziehungsweise als dessen Gegenstück im Rahmen des familialen Dreiecks. Die Feindschaft gegenüber dem Vater liege darin begründet, dass Medea nicht die Stelle der Mutter einnehmen dürfe. Weil sie nicht libidinöses Objekt des Vaters werden könne, den sie begehre, seien sowohl der Geschlechterkampf mit Jason als auch die Kindermorde Symptome einer narzisstischen Kränkung, welche die weibliche Kindheit überschatte und die libidinösen Objektbeziehungen der erwachsenen Frau präge. Wenn die Identifikation mit der ,guten` Mutter nicht gelingt, dann kann dieser Medeakomplex nicht ,untergehen':

In allen Verbrechen, die wir gerade besprochen habe, ist die väterliche Figur präsent; und meistens als Kontrapunkt eine andere Figur. Sie kann als Objekt betrachtet werden, mit dem das Subjekt, in diesem Falle Medea, sich in einer Position der Rivalität, der Eifersucht und der Feindseligkeit befindet: Rivalität zwischen Medea und Jason und, im äußersten Fall, zwischen Medea und ihren Kindern. (Bécache 1982, 786; Übers. F. B.)

Diese ,tabuisierte Verknüpfung zwischen Bewußtsein und weiblicher Aggression“ mache Euripides' Medea als „tragisches Subjekt“ (Hidalgo-Xirinachs 2002, 239) zum Gegenstück des sophokleischen Ödipus, das im Kindsmord Agency erhält; Selbstbestrafung und Selbstopferung bewertet Roxana Hidalgo-Xirinachs daher als Reaktion auf diesen autonomen Akt (vgl. Hidalgo-Xirinachs 2002, 257-271). 
Im Gegensatz zur Rolle des Vaters betont daher Marianne Leuzinger-Bohleber die Rolle der Mutter in der ,gesunden' Entwicklung der weiblichen Psyche. MedeaPhantasien analysiert sie bei Frauen mit psychogener Frigidität und Sterilität (vgl. Leuzinger-Bohleber 1996 u. ö.). Aufgrund gestörter frühkindlicher Beziehungen, die durch Depressionen der eigenen Mütter bedingt seien, verbinden diese Frauen sexuelle Lust mit der Angst vor der Abhängigkeit beziehungsweise des Verlusts ihrer Liebespartner, sodass sich ihre weibliche Identität durch ein hohes Maß an Ambivalenz auszeichne. Dabei verschiebt Medea die eigene Traumatisierung auf die Kinder, deren Mord Sonia Saporiti in Beziehung zu Freuds Jenseits des Lustprinzips (1920) setzt und als Wirkung des Todestriebes bewertet (vgl. Saporiti 2013, 115-122). Das Potenzial von Generativität sowie - gleichzeitig - die Destruktivität von Generativität erzeuge die Imago des mütterlichen Körpers als eines für die ,Kinder‘ bedrohlichen, todbringenden Innenraumes (vgl. Irigaray 1989 [1981]; Mauerer 2002, 57-97). Dieses Potenzial richte sich, perspektiviert Hidalgo-Xirinachs, vor allem gegen Kreusa. In einer Metonymie verbindet sie vor dem Hintergrund von Melanie Kleins Frühstadien des Ödipuskomplexes (1927) den weiblichen im mütterlichen Körper verborgenen Phallus, die beiden Kinder sowie Medeas Gabe zu einer Metonymie des Begehrens nach der Mutter und der Bedrohung durch sie - umgekehrt folgt daraus: „Die mütterliche Wut kehrt gewalttätig zurück, um den jungen, begehrenden und begehrten Körper der adoleszenten Tochter zu zerstören.“ (Hidalgo-Xirinachs 2002, 279) Eine dergestalt anal-sadistisch grundierte Triangulierung sei für die Phantasien einer frühen Triangulierung typisch, sodass der Mord an Kreusa die Urszene nachstelle.

In ihrer Interpretation der Tragödie löst sich Riitta Sirola von der psychoanalytischen Tradition, Medea als erwachsene Frau zu behandeln. In Anlehnung an Ernest Jones' Symbolbegriff ( $\rightarrow$ II.1. SEMIотIK) interpretiert sie Medea als paradigmatische Figur für eine bestimmte Phase in der psychischen Entwicklung. Gegenüber der ödipalen Triade macht Sirola indes die narzisstische Dyade stark, die in dieser Phase das Seelenleben von Mädchen bestimme. Die Medea-Konfiguration bilde dementsprechend ein präödipales Stadium ab, das jedes Mädchen auf dem Weg zu einer gelungenen weiblichen Geschlechtsidentität durchlaufe: „In my view, the myth can be seen as a dramatisation of a little girl's dependence on her mother. [...] I tend to interpret the myth from the point of view of impulses both enabling and preventing a girl's pre-oedipal development. The myth represents a journey of a girl's desire (libido) from the beginning of her life towards the oedipal configuration.“ (Sirola 2004, 100)

Diese Entwicklungsphase zeichne sich durch eine psychische Ambivalenz von Bindungswünschen und Trennungsbestrebungen aus, die nur eine starke dritte Position auflösen könne: „Who could - as happens in reality - pull the girl child out of the magic circle of her magical mother's realm? Who else but a man - 
the father." (Sirola 2004, 101) In Euripides' Medeia repräsentiere Hekate, die dunkle Göttin der Magie, Theurgie und Nekromantie eine dergestalt magische, überlegen-starke und deshalb phallische Mutter, die solche Ambivalenzkonflikte hervorrufe. Weil Kreon und Aigeus als präödipale, das heißt in ihrer Sexualität bedrohliche Väter in Erscheinung treten, sei die symbolische Position des Vaters, der die Hekate-Imago neutralisieren könne, nicht besetzt, sodass der Medeakomplex eben nicht untergehen könne. Auch der schwache Jason besetze nicht die symbolische Position des postödipalen Vaters, sondern fungiere als Agent der Mutterimago. In narzisstischer Doppeleinheit mit Jason wiederhole Medea daher die Mutterbeziehung. Liebesgeschichte und Kindermorde spiegeln die Entwicklung eines kleinen (noch) narzisstischen Mädchens wider: „A pre-oedipal girl has as passionate control over every interest or urge from her mother that is not directed to her, seen in the hight of envy towards, e. g., penis or sisters or brothers. If the dyadic attachment becomes obscured, it exposes the girl to fury with the ultimate goal of restoration of the dyad at all costs. Then all third elements that threaten the dyad will be eliminated." (Sirola 2004, 103) Indem Jean Anouilh den Konflikt zwischen Jason und Medea in seiner Médée (1946) als Geschlechterkampf inszeniert, bestätigt er Sirolas Interpretation. Mit dem Entschluss, ihre Kinder zu töten, verwandelt sich Medea in das Mädchen zurück, das sie ist: „Ich habe die Unschuld zu schlachten noch in diesem kleinen Mädchen, das so viel gewollt hätte, und in diesen beiden kleinen lauen Stücken von mir." (Anouilh 1946, 401; Übers. F. B.) Für Hélène Cixous ist Medea deshalb das Paradigma der Ortlosen: „Where to stand? Who to be?“, fragt sie ortlos in der Erkenntnis, „that total love has been used by the loved one for his base ambitions“ (Cixous [1975] 1986, 75).

Die präödipale Konfiguration erlaubt freilich noch eine andere Interpretation, in der die Mutterimago erstens figurenperspektivisch von Medea getrennt und die Figur zweitens mit dieser Imago in Beziehung gesetzt wird; Medea hat dann keine ambivalente Mutterimago mehr, sondern ist Bestandteil einer solchen (vgl. Balter 1969, 249-259). Im 18. Jahrhundert erhalten die Kinder sprechende Rollen und mit ihnen die Aufgabe, vor der Fremden, die ihre Mutter ist, Angst zu haben. Klinger räumt dieser Angst der beiden erstmals namentlich genannten Söhne Memeros und Feretos den grandiosen vierten Akt ein, und Grillparzer lässt einen der beiden die Mutter fragen: „Ich fürchte mich. [...] Tust du mir nichts?“ (Grillparzer 1986 [1822], 378) Medeas Sexualität und Aggressivität steigern sich bis zu Hans Henny Jahnns schwarzer, alter, geiler Medea (1926/1959) - einer Allegorie der beiden elementaren Triebe Eros und Thanatos, die Jahnn durch eine krude Sexualsymbolik (Pferd) sowie die inzestuösen Familienbeziehungen (Vater - Söhne, Mutter - Söhne, älterer Sohn - jüngerer Sohn) bebildert. Diese Imago zeugt von einer präödipalen Angst vor der Mutter. Im Rahmen seiner 
Abhandlung Über die weibliche Sexualität (1931) erkennt Freud, dass die Angst des männlichen Kindes, „,von der Mutter umgebracht zu werden“, vom mütterlichen Ursprung oft - sekundär - auf den Vater verschoben wird, der dann wie Saturn seine Söhne frisst: „Die Angst, gefressen zu werden, habe ich bisher nur bei Männern gefunden, sie wird auf den Vater bezogen, ist aber wahrscheinlich das Verwandlungsprodukt der auf die Mutter gerichteten oralen Aggression. Man will die Mutter auffressen, von der man sich genährt hat; beim Vater fehlt für diesen Wunsch der nächste Anlaß.“ (XIV, 531)

Doch nicht nur Medeas Söhne, sondern vor allem auch den König von Korinth zeichnet in allen Medea-Bearbeitungen seit Euripides eine auffällige Ambivalenz gegenüber Medea aus. Anouilh schildert Kreon schließlich in einer Regression, die ihn völlig handlungsunfähig macht (vgl. Anouilh 1946, 378) Mit dieser Abwehr, die sich in der Angst vor Medea ausdrückt, rückt ein männliches präödipales Bewusstsein ins psychoökonomische Zentrum der Medea-Konfiguration. In ihrer Monstrosität repräsentiert Medea den Archetypus einer prima materia am unbewussten Grund der Seele. Dort liegt deren Anderes, mit dem sich das männlich indizierte (Selbst-)Bewusstsein zu verbinden trachtet, um den Zustand seelischer Vollkommenheit zu erreichen. Im Mysterium Coniunctionis (1954) weist Carl Gustav Jung diesen Archetypus in Bezug auf Karl Kerényis religionswissenschaftliche Studie Die Töchter der Sonne (1944) in alchemistischen Traktaten nach, in denen er solche psychischen Entwicklungsprozesse bebildert findet: Bei der weiblichen Wandlungssubstanz (prima materia) ,handelt es sich um eine typische Kombination der Motive von Liebe, Heimtücke, Grausamkeit, Mütterlichkeit, Verwandten- und Kindsmord, Zauber, Verjüngung und - Gold“ (Jung 1972 [1954], 20-21).

\section{Zwischen Matriarchat und Patriarchat}

Was Jung als Archetypus bezeichnet, würde ich etwas neutraler als Repräsentation des kulturellen Imaginären verstehen wollen. In den Medea-Bearbeitungen verweist es auf einen Bereich, den die erste Tragödie ausgeschlossen hat. Denn dem Ausgeschlossenen entkommt man nicht; es wirkt in der Latenz. Vor diesem Hintergrund führt David Pister seine Euripides-Lektüre von einem individualpsychologischen zu einem kulturimagologischen Modell. Anstatt den Figuren gilt seine Aufmerksamkeit der Struktur und den Funktionen der Kindermorde. Sie stellen „weniger eine unfassbare Gräueltat dar, sondern erweis[en] sich bei genauerem Hinsehen wiederum als das Medium, mit dem Medea aus dem Dazwischen an einen eindeutigen gesellschaftlichen Ort gelangen kann“ (Pister 2013, 
141). Morde im Allgemeinen initialisieren Medeas geographische Bewegung von Ort zu Ort sowie ihre soziale Bewegung von Oikos zu Oikos (Haus- und Wirtschaftsgemeinschaft). Die Kindermorde im Besonderen setzen diese Bewegung fort, weil sie Medea von Korinth nach Athen und damit verbunden von Jasons Oikos in denjenigen des Aigeus führen. Pister kommt zu dem Ergebnis, dass Medea eine transgressive Figur ist, was nicht zuletzt durch ihre konstitutive Ambivalenz bestätigt wird: Etymologisch verweist der Name sowohl auf medos, Rat und Sorge, als auch auf einen Anschlag; medea maches bezeichnet den Schlachtplan (vgl. Pape 1884, 910-911). Euripides führt Medea als unheimliche Melancholikerin ein (vgl. Euripides 1972 [431 v. Chr.], 91), sodass sie im Horizont antiker Humoralpathologie dadurch sowohl in die Nähe von Kranken als aber auch von ausgezeichneten Dichtern, Philosophen und Staatsmännern rückt. Zwischen ,Männlichkeit‘ und ,Weiblichkeit' ist auch Medeas kulturelles Geschlecht angelegt (vgl. Pister 2013, 146-148). Vor allem aber steht Medea im kulturellen Imaginären zwischen der Göttin Hekate, der sie als Priesterin dient, und ihrem Großvater Helios, auf dessen Schlangenwagen Euripides die Heliade am Schluss der Tragödie entkommen lässt. Kosmologisch bewegt sie sich demzufolge zwischen den Sphären des Mondes und der Sonne. Deshalb führt Medeas Bewegung, so wie Johann Jakob Bachofen in seiner Studie Das Mutterrecht (1861) spekuliert, vom lunarischen Tellurismus zum Solismus.

Gesine Palmer ergänzt, dass die Tragödie auf einen soziokulturellen Funktionszusammenhang verweist. Aufgrund ihrer göttlichen Abstammung widerstreiten in Medea Ehrgefühl und Muttergefühl. Während die menschlichen Frauen „im Regelfall des Erdenlebens als Geiseln des Muttergefühls der Willkür der Männer ausgeliefert bleiben, wenn sie nach Durchsetzung der Patrilokalität ihre Kinder im Haus des Mannes großziehen wollen“ (Palmer 2006, 79), bewegt sich Medea zwischen Matrilokalität und Patrilokalität. In Euripides' Medeia, so führt Palmer aus, lassen sich daher die Spuren einer alternativen Gründungsgeschichte des Patriarchats nachzeichnen. Am Beginn der Ablösung des Matriarchats stünde kein Vatermord - „(diese Vorstellung gehört ganz ins Innere einer patriarchalischen Weltordnung, in der Frauen nicht mehr als Agierende, sondern nur noch als Belohnung, um die gestritten wird, vorkommen)“-, sondern ein Kindsmord: „Eine moralische Lehre aus diesen Erzählungen scheint zu besagen: Damit das nicht wieder vorkommt, müssen, von nun an` Frauen von Männern dazu gezwungen werden, ihre Kinder oder die Kinder der Rivalin nicht zu töten, sondern zu versorgen.“ (Palmer 2006, 79)

Der durch die Kindermorde motivierten Bewegung der Figur auf der syntagmatischen Ebene der Tragödie liegt also eine Bewegung auf der paradigmatischen Ebene zugrunde, die ad fontes führt. Denn hinter Medeia treten die inkommensurablen Züge einer aphroditisch-hetärischen Mutterimago hervor, die auf einen 
älteren, chthonisch-maternalen Bereich zurückgeht. Wie in Sophokles' Oidipous Tyrannos (429-425 v. Chr.) widerstreiten auch bei Euripides zwei Ursprungsgeschichten in einer Konfiguration ( $\rightarrow$ III.6. ÖDIPUS), die dadurch die Schwelle von ,Alt' und ,Neu' bildet: Ist der Mensch aus der Erde geboren, also chthonischen Ursprungs, oder stammt er von biologischen Eltern ab; ist er also aus ,einem entstanden, oder ist er von zweien gezeugt worden? Und wie der Ödipus-Mythos basiert auch der Medea-Mythos auf einer doppelten Struktur, in der kosmologische und biologische Genealogie einander im Widerstreit absichern: Autochthonie wird verneint (Helios) und bejaht (Hekate) sowie Verwandtschaft abgewertet (Kindermord) und aufgewertet (Mutterliebe) (vgl. Lévi-Strauss 1967 [1958], 226-254). In seiner autorzentrierten Interpretation, dass Medeia Ausdruck des Wunsches nach poetischer Autonomie ist, macht sich Bennett Simon eben diesen Aspekt der Autochthonie für Euripides’ Kopfgeburt zunutze: „Following through with this metaphor, the play, the children, are hybrids that cannot survive youth, or sterile hybrids. Or we might invoke the possibility that the wishes for unisexual reproduction in Medea [...] represent his fantasy, his wish, his perception of himself as thoroughly unique in conception and birth.” (Simon 1988, 100)

In seiner Tragödie verdeckt Euripides den chthonisch-maternalen Bereich buchstäblich. Lediglich ein einziges Mal ruft Medea Hekate an, die nicht von ungefähr als Göttin der Wegkreuzungen, Schwellen und Übergänge und Wächterin der Tore zwischen den Welten gilt und somit das Relais bildet, das vom mythologischen Syntagma auf das Paradigma umschaltet:

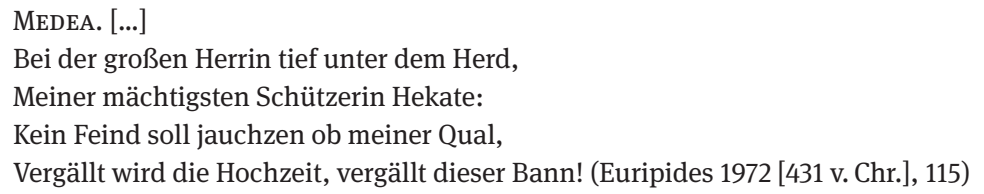

Die Verbindung zwischen Medea und Hekate ist indes keine genealogische Frage - als ,reale "Mutter werden in den Quellen alternativ die Okeanide Idyia, die Nymphe Neara oder Eurylyte gehandelt, die Gattin von Medeas Vater Aietes (vgl. Corbineau-Hoffmann 2008). Auch der Umstand, dass Medea in späteren Bearbeitungen der Hekate als Priesterin dient, ist gegenüber der Tatsache zweitrangig, dass Euripides mit der Medea-Hekate-Quellenverbindung eine symbolische Position im kulturellen Imaginären markiert. Apollonius von Rhodos stellt in der Argonautica (3. Jh. v. Chr.) darüber hinaus eine Verbindung zwischen Medea und der Mondgöttin her (Titanin) (vgl. Apollonius 1996 [3.Jh.v. Chr.], 82-83). Doch lassen sich im chthonisch-maternalen Bereich die Göttinnen ohnehin nicht mehr scharf voneinander trennen. Euripides referiert beispielsweise auf Themis und 
Artemis, mit denen Hekate in der Mythologie eine Dreieinheit bildet (vgl. Euripides 1972 [431 v. Chr.], 101).

Dass Medea eine Eklipse dieser mater magna ist, lässt sich einerseits religionswissenschaftlich mit der Geschichte der Medea-Kulte belegen (vgl. CorbineauHoffmann 2008). Andererseits rückt sie durch den Verjüngungszauber, mit dem Medea Macht über Leben und Tod hat, selbst in die Nähe der chthonisch-maternalen Göttinnen, die Leben geben und nehmen. Die Grausamkeit des Rituals Medea zerstückelt die Menschen und kocht sie in ihrem Kessel - betont noch einmal den dunklen Hinter- und Abgrund der Figur. In diesem Sinne wartet Apollonius von Rhodos mit einer Geschichte auf, in der statt des Sonnengottes die drei großen Göttinnen triumphieren, die nicht zuletzt auch die Verantwortung für den Trojanischen Krieg tragen. Hera, Athene und Aphrodite (Kypris) treiben ihr Spiel mit den Menschen: „Auf, laß uns zu Kypris gehen! Wir wollen uns beide an sie wenden und sie auffordern, ihrem Sohn - sofern er folgsam ist - zu sagen, er solle die zauberkundige Tochter des Aietes mit seinen Geschossen treffen und in Liebe zu Jason entbrennen lassen.“ (Apollonius 1996 [3. Jh. v. Chr.], 3) Für Kerényi verdeckt diese harmlose Geschichte ebenso wie der Verjüngungszauber lediglich die grausamen Züge der aphroditisch-hetärischen Mutterimago (vgl. Kerényi 1944, 91-116).

Um eine Verdeckung handelt es sich meines Erachtens auch bei dem invarianten Faktum des doppelten Kindermordes. Andere Quellen weichen sowohl bei der Zuschreibung der Morde an Medea, insbesondere aber bei der Anzahl der ermordeten Kinder, die sich ins Unrealistische verliert, erheblich von Euripides ab: Zwei, sieben, zehn - viele geschlechtlich unbestimmte Kinder tötet Medea (vgl. Lesky 1931, 42-43). Wenn aber vielen Kindern der Tod droht, dann als Pars pro Toto allen Menschen. In der Sache - und auf der Basis der Analogie, die von der Psychoanalyse zur Kulturanalyse führt - handelt es sich bei Euripides' Korrektur um eine Verschiebung. Der rational und emotional ganz problemlos zu bewältigende Mord an genau zwei Söhnen auf der einen Seite sowie die Personalisierung dieser Bedrohung durch die leibliche Mutter auf der anderen fungieren als kollektive Deckerinnerung der allgegenwärtigen Bedrohung des Lebens durch den Tod, die das Trauma der Kultur ist. Solche Verschiebungen beschreibt Freud in der Psychopathologie des Alltagslebens (1904) individualpsychologisch folgendermaßen:

Da es bekannt ist, daß das Gedächtnis unter den ihm dargebotenen Eindrücken eine Auswahl trifft, stände man hier vor der Annahme, daß diese Auswahl im Kindesalter nach ganz anderen Prinzipien vor sich geht als zur Zeit der intellektuellen Reife. Eingehende Untersuchung weist aber nach, daß diese Annahme überflüssig ist. Die indifferenten Kindheitserinnerungen verdanken ihre Existenz einem Verschiebungsvorgang; sie sind der Ersatz in der Reproduktion für andere wirklich bedeutsame Eindrücke, deren Erinnerung 
sich durch psychische Analyse aus ihnen entwickeln läßt, deren direkte Reproduktion aber durch einen Widerstand gehindert ist. Da sie ihre Erhaltung nicht dem eigenen Inhalt, sondern einer assoziativen Beziehung ihres Inhaltes zu einem anderen, verdrängten, verdanken, haben sie auf den Namen ,Deckerinnerungen', mit welchem ich sie ausgezeichnet habe, begründeten Anspruch. (IV, 51)

An der Schwelle von Matriarchat zu Patriarchat leistet Euripides mit seiner Tragödie also ästhetische Traumatherapie, deren Energie bis heute die kulturelle Arbeit am Mythos in Gang hält - eine Arbeit, die man als Bewirtschaftung der Grenze bezeichnen kann. Im Grundsatz basiert diese Arbeit auf einer Subversion der Darstellung. Denn das, was jenseits der Grenze vermutet wird, schließt das aus, was diesseits von ihr liegt, indes wird die Differenz von Diesseits und Jenseits überhaupt erst begründet. Ernesto Laclau setzt unter anderem in Emancipation(s) (1996; Emanzipation und Differenz) für diese Subversion einen leeren Signifikanten voraus: „Aber wenn wir über die Grenzen eines Bezeichnungssystems sprechen, ist klar, daß dessen Grenzen nicht selbst bezeichnet werden können, sondern sich selbst zeigen müssen als die Unterbrechung oder der Zusammenhang des Prozesses der Signifikation.“ (Laclau 2002 [1996], 66)

Als leerer Signifikant ,zeigt" also auch Medea vom Diesseits auf das Jenseits der Grenze, ohne diesem Bereich indes eine Anschauung zu unterlegen. Die Stelle des Signifikats nimmt vielmehr die Enzyklopädie ein, sodass sich die Bedeutung des leeren Signifikanten gewissermaßen im Nichts der Entropie verliert. In seinen Mythologies (1957; Mythen des Alltags) beschreibt Roland Barthes den Mythos in diesem Sinn als „sekundäres semiotisches System“: „Alles verläuft so, als verschöbe der Mythos das formale System der ersten Bedeutungen um eine Raste.“ (Barthes 2010 [1957], 258) Auf ihre eigentliche Leere referieren alle Medea-Bearbeitungen in der Regel mit der metafiktionalen Strategie ausgestellter Selbstreflexivität. Immer weiß Medea genau, wer oder was sie ist, nicht ist, war, sein wird, sein soll oder möchte - Medea eben: „NUTRIX: Medea ... / MedEA. Fiam.“ (Seneca 1961 [um 45 n. Chr.], 252) - „Ich bin Medea, bin nicht eure Mutter.“ (Klinger 2012 [1786], 65) - „Medea bin ich wieder, Dank euch Götter!“ (Grillparzer 1986 [1822], 374) - „Ich bin endlich Medea, für immer.“ (Anouilh 1946, 402; Übers. F. B.) Nur keiner weiß, was ,Medea‘ eigentlich bedeutet!

\section{Serialität: Dea Lohers Manhattan Medea (1999)}

Es ist diese Leerstelle, in der das ausgeschlossene kulturell Unvordenkliche und individuell Unbewusste imagologisch zusammenfallen. Als Katalysator hält sie die Arbeit am Mythos in der Literatur und anderen Medien im Lauf der Zeit 
ebenso in Gang wie die psychoanalytischen Interpretationen dieser Arbeit. In Abhängigkeit historischer Darstellungsmedien und -formen, folgen alle MedeaBearbeitungen der Wiederholung des Traumas, auf das der leere Signifikant ,Medea' verweist, das er aber nie (er-)füllt. In der Postmoderne haben sich die Rahmenbedingungen für diese ästhetische Traumatherapie radikal verändert. Grillparzer reichen noch Medeas Hände, mit denen sie symbolisch für ihre koloniale Vergewaltigung die griechische Leier zerbricht, Jahnn setzt alles auf die Karte des chthonisch-maternalen Symbols des Pferdes, um Medea eine Anschauung zu unterlegen. Vor allem der Film macht Medea in der zweiten Hälfte des 20. Jahrhunderts zu einem regelrechten screen. Die drei großen Medea-Verfilmungen von Pier Paolo Pasolini (1969), Lars von Trier (1988) und Natalia Kuznetsova (2009) nutzen im Abstand von je zwanzig Jahren die filmischen Mittel der Schnitttechnik (long takes), der multiplen Projektion und des Sounds, um die Dialektik von Mythos und Geschichte, von Bild und Affekt sowie von Sichtbarem und Hörbarem auf Medea zu projizieren. An die Stelle der erfüllten Repräsentation tritt dabei die leere Form selbst (mein Dank für dieses Argument gilt Lutz Koepnick).

Im Medium der Sprache greifen insbesondere die literarischen Medea-Bearbeitungen zu drastischen Mitteln, um der referenziellen Funktion der Sprache zu entkommen und die Semantik der Form zu nutzen. So verbindet Heiner Müller in seinem literarischen Triptychon Verkommenes Ufer Medeamaterial Landschaft mit Argonauten (1982) einzelne Lexeme zu einer bedeutungsoffenen Reihe, so unter anderem: „Schlamm“, „Kothaufen“, „FROMMS ACT“ (Kondom-Marke), „CASINO“ (Zigaretten-Marke), „Monatsbinden“, „Blut“, „Weiber von Kolchis“, „SCHLAMMFOTZE“, „STOSS MICH KOMM SÜSSER“ (Sexualakt), „Abflußrohre, Kinder ausstoßend in Schüben gegen den Anmarsch der Würmer“, „Beischlaf in Chikago“, „Blutbeschmierte Weiber“, „Leichenhallen“, „Tote[ ]“, „Abort“, „beschissen[e] Erde“ (Müller 2002, 73-74). Die Serie konfiguriert das Aphroditisch-Hetärische. Durch Anspielung auf die Wehrmachtsverbrechen: „EINIGE HINGEN AN LICHTMASTEN ZUNGE HERAUS VOR DEM BAUCH DAS SCHILD ICH BIN EIN FEIGLING“ verdichtet Müller die Konfiguration zu einer geschichtsphilosophischen Allegorie der westlichen Kultur, welcher der leere Signifikant ,Medea‘ buchstäblich zugrunde liegt:

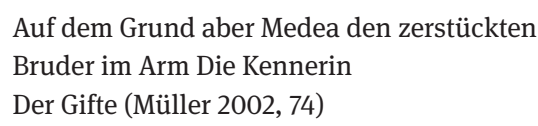

Auf dem artifiziellen Weg, der Inhalt durch Form ersetzt, entscheidet sich Dea Loher für Verfahren der Metatheatralität (vgl. Haas 2006, 259), um die Semantik der Form für die Füllung der Leerstelle in den Dienst zu nehmen. In ihrem 
Migrationsdrama Manhattan Medea (1999) stammen Medea und Jason (englisch auszusprechen) aus dem Kriegsgebiet des ehemaligen Jugoslawiens. Als illegale Einwanderer leben die Mazedonier - mit diesem Anachronismus beugt Loher jedem mimetischen Missverständnis vor - im historischen Immigrantenviertel Alphabet City in Manhattans East Village. In typologischer Beziehung zum euripideischen Prätext stellt Loher den beiden Protagonisten in der Rolle des Königs Kreon den Boss eines sogenannten Sweat-Shops gegenüber - einer Färberei, die menschliche wie natürliche Ressourcen ausbeutet. Seine schöne Tochter Claire alias Kreusa - lässt er an der Juilliard School Geige studieren; und für ihre geplante Hochzeit mit Jason bringt der Vater wenig Begeisterung auf. Wie Kreon schiebt auch der Sweat-Shop-Boss Jasons fremde Exfrau einfach ab. Den Kindermord motiviert Loher daher nicht nur psychologisch, sondern auch geo-, ethno-, wirtschafts- und sozialpolitisch. Medea will ihren einzigen Sohn nicht dem Vater und seiner schönen, reichen, neuen Braut von der Fifth Avenue überlassen. Wie Müller setzt auch Loher auf die poetische Funktion der Sprache: Ambiguierung der Syntax (u.a. durch fehlende Satzzeichen), Rhythmisierung, Metaphorisierung, wobei der traumatisierte, taube Transvestit Deaf Dasy als Medium mythopoetischen Sprechens, vor allem aber der ,Doorman“ Velazquez wichtige Funktionen innehaben: „Mein Name ist Velazquez. [...] Ein falscher Velazquez, der ein echter ist.“ (Loher 1999, 10-11) Indem er sein Leben dem Kopieren der Gemälde seines berühmten Namensvetters, des spanischen Malers Diego Velázquez widmet, fungiert die Figur als selbstreflexives Medium des leeren Signifikanten.

In der neunten Szene schaltet Loher von Mimesis auf Semiosis, das heißt von Inhalt auf Form um. Die Szene beginnt damit, dass der ,Doorman` Medea ihren Sohn bringt, damit die Mutter sich von ihrem Kind verabschieden kann. Dabei überreicht er ihr eine seiner Velázquez-Kopien als Geschenk.

Das Bild zeigt „Philipp Prosper. 1659“ (Loher 1999, 60), wie man aus einer Fußnote erfährt: Sohn des spanischen Königs Philipp IV. und Anna Marias von Österreich. Der Junge ist im Alter von vier Jahren gestorben. Indem nun Medea das Bild aus der Mülltüte nimmt und den Sohn in der Mülltüte erstickt, stellt die Szene eine metaphorische Substitution (Verdichtung) zur Schau: In der Mülltüte nimmt der Junge buchstäblich den Platz des toten Prinzen ein. Mit dem Mord Medeas an ihrem Kind folgt Loher ebenso Euripides wie mit dem anschließenden Mord an Jasons Braut Claire. Deaf Daisy überbringt Claire Medeas tödliches Geschenk - ein aus der väterlichen Gerberei verseuchtes rotes Lederkleid, das Claires Haut verätzt, sie also gewissermaßen verbrennt. In metonymischer Kontiguität (Verschiebung) würde Claire auf der Bühne tatsächlich lichterloh zu brennen haben, wie im Nebentext der Szene beschrieben wird - in einem Nebentext, der übrigens auffällige Ähnlichkeiten zu Doras erstem Traum aufweist, den Freud analysiert: „In der Tür des Hauses erscheint eine Fackel, menschengroß, 
lodernd. Man hört sonst kein Geräusch. Keinen Schrei. Nur Stille. Das Feuer greift auf Velazquez’ Gemälde über. Solange die Fackel brennt, singt Deaf Daisy. Mit ihrer Stimme erstirbt auch das Feuer. Anstelle des ursprünglichen Bildes kann man nun Picassos ,Las Meniñas“ erkennen.“ (Loher 1999, 61)

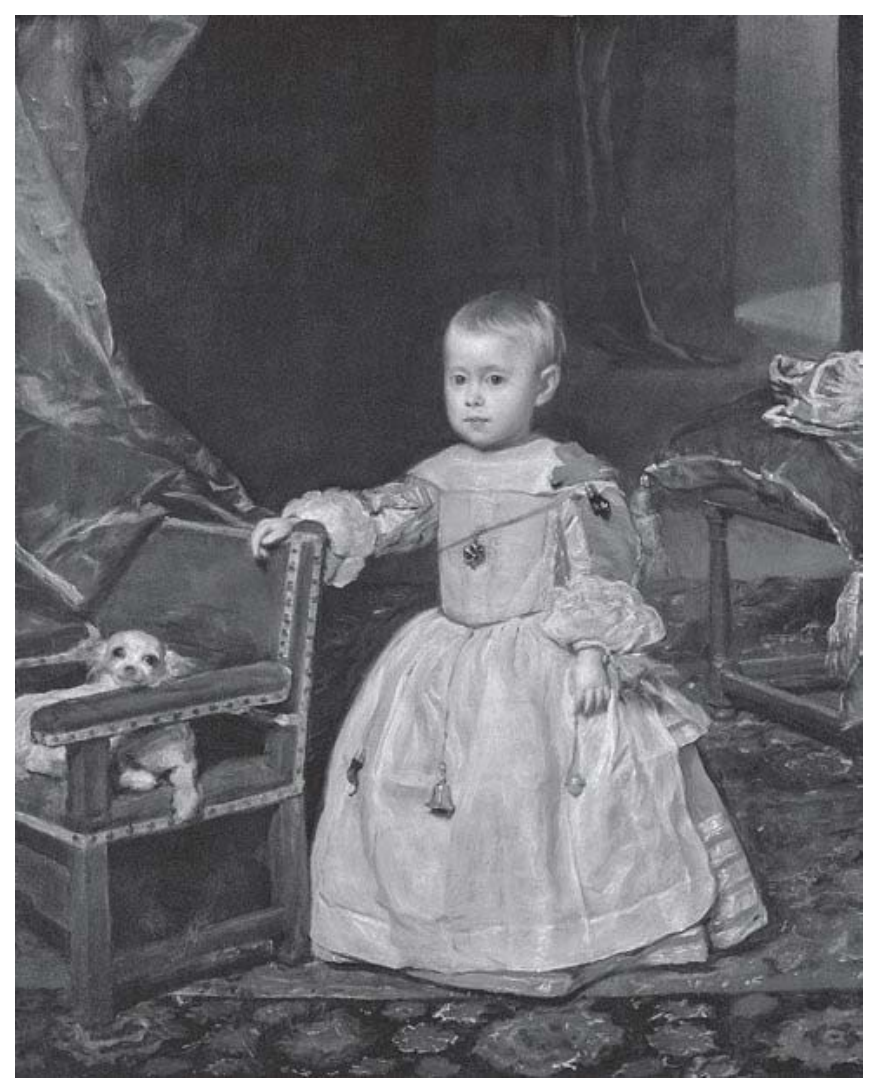

Abb. 1: Velázquez, Diego. Infant Philipp Prosper. 1659. Wien. Kunsthistorisches Museum Wien, Gemäldegalerie.

Beide Morde, die nicht dramatisch unmittelbar, sondern narrativ mittelbar dargestellt werden, verbindet eine gemeinsame Symbolik: Sowohl die Schwelle unterstrichen durch die beiden Schwellenfiguren des ,Doormans ${ }^{\text {‘ }}$ und des Transvestiten - als auch die Fackel sind Attribute der Hekate; ihr Feuer brennt ebenfalls unter Medeas Kessel der Wiedergeburt. Durch das Feuer entsteht daher die Quellenverbindung Hekate-Medea, als deren Priesterin Deaf Daisy ein Wiedergeburtsritual begleitet: Das Feuer zerstört das Gemälde von Felipe Próspero, 
bis man an dessen Stelle ein Bild von Picasso erkennen kann. Wie bei dem ersten Bild handelt es sich auch bei diesem zweiten um eine Kopie des Originals. Doch nicht etwa der ,Doorman', vielmehr Pablo Picasso experimentiert 1957 in nicht weniger als achtundfünfzig Variationen mit Velázquez’ berühmtem Gemälde Die Hoffräulein (1656) (vgl. Picasso 1960). Es bildet Margaríta Teresa Maria - die den Bruder überlebende Schwester Felipe Prósperos - im Kreise von Dienerinnen und Zwergen ab.

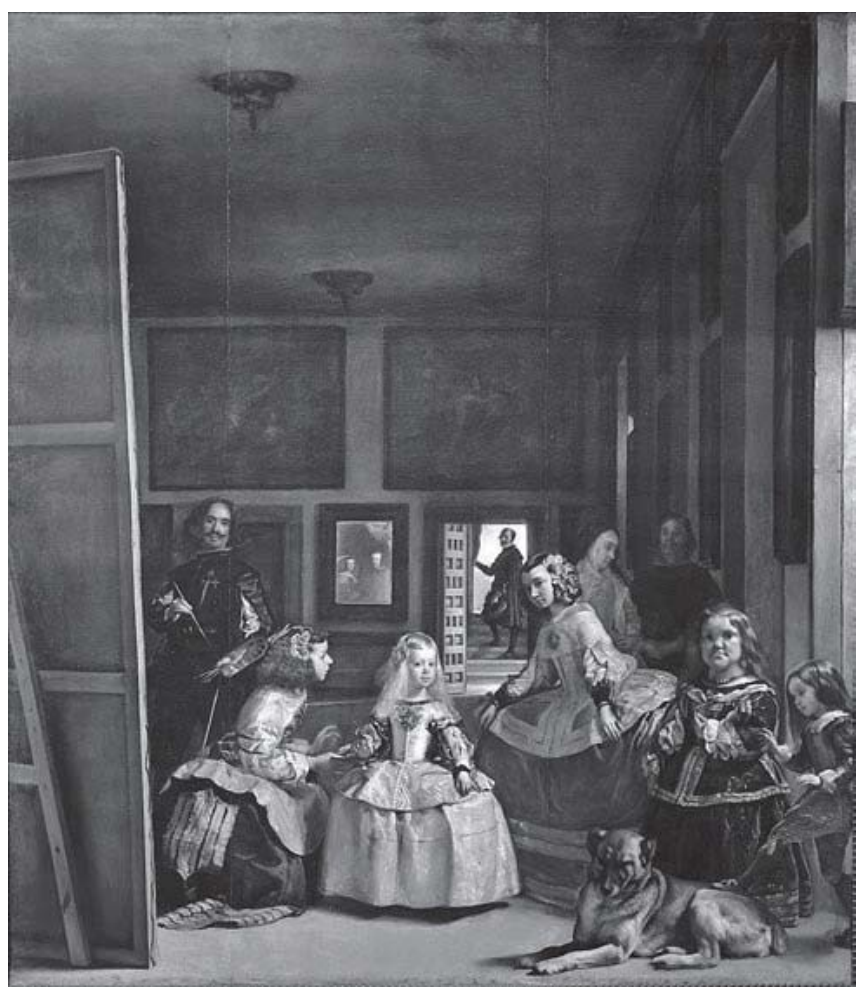

Abb. 2: Velázquez, Diego. Las Meninas. 1656. Madrid. Museo del Prado.

Wenn nun aber Felipe für Medeas ermordeten Sohn steht, dann Margaríta für das in dieser Medea-Bearbeitung so offenkundig fehlende zweite Kind. Weil nämlich das Mädchengemälde die Stelle des Knabengemäldes einnimmt, ersetzt Medeas Tochter Jasons Sohn, dessen Opfer die Voraussetzung dieser symbolischen, intermedial inszenierten Geburt ist. „Sich selbst zurückgeben, ist die einzige Lösung. Deshalb das Bild“ (Hörnigk 1999, 6), so kommentiert die Autorin. Mit den beiden Geschwistern korrigiert Loher also ganz offenkundig die mythologische Genealo- 
gie, in der nicht etwa die Spur der Jasoniden getilgt wird. Diese Genealogie kommt tatsächlich ganz ohne Väter und Söhne aus. Dergestalt sprengt die maternale Genealogie die lineare Abstammungsfolge und schließt sich zu einem weiblichen Zyklus, in dem Hekate, Medea und ihre Tochter als austauschbare Variablen an ein und derselben symbolischen Position in Erscheinung treten.

Bereits Velázquez' „ironische Leinwand“ (Foucault 1971, 35) ist bekanntermaßen hochgradig medienreflexiv, weil das Original die Bedingungen der Möglichkeit von Repräsentation an und für sich ausstellt. Als Repräsentationen der Repräsentation steigern die Kopien des ,Doormans ' diese Ironie, die wiederum Picasso mit seiner Serie an den Ursprung ihrer konstitutiven Leere führt: Als Kopie der Kopie der Kopie der Kopie verschwindet das vermeintlich Repräsentierte nämlich buchstäblich von Picassos Bildfläche und wird immer und jedes Mal durch etwas anderes ersetzt. Weil in Lohers Nebentext indes nicht erwähnt wird, in welches Bild sich das ,ursprüngliche‘ eigentlich verwandelt, weil der zitierte Titel also metonymisch auf alle Bilder der Serie zugleich verweist und nicht nur auf das Bekannteste, erhält Las Meninas - als Metonymie - den Status eines leeren Signifikanten.

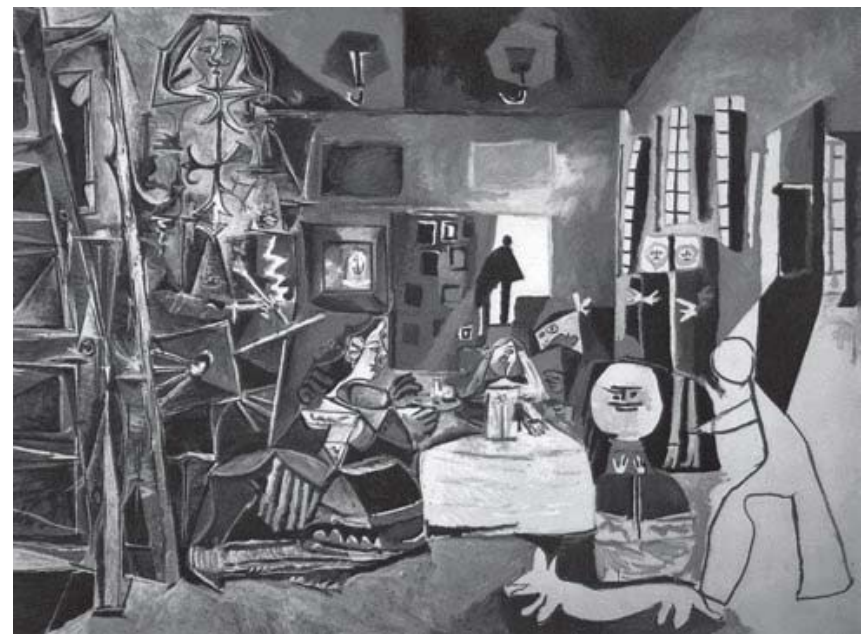

Abb. 3: Picasso, Pablo. Las Meninas. 1957. Barcelona. Museu Picasso.

Mit dem Verweis auf den Bildtitel unterlegt Loher ihrer zyklischen Genealogie deshalb eben gerade keine Anschauung, sondern konfiguriert stattdessen eine mise en abyme. Mit den Bildzitaten macht sie sich für ihre Arbeit am Mythos also das Reflexionspotenzial zunutze, das zwischen dem Text und der Serie entsteht, um die Leerstelle in Manhattan Medea im Medium des dramatischen Textes 
semantisch zu füllen. Dabei kommt sie dem chthonisch-maternalen Bereich, in dem Leben und Tod in der Macht einer großen Göttin stehen und den Euripides so entschieden ausgeschlossen hat, für einen Augenblick ganz nahe, bevor die Arbeit am Mythos weitergeht.

\section{Literatur}

Anouilh, Jean. „Médée“. Anouilh, Jean. Nouvelles pièces noires. Paris 1946: 355-403.

Apollonius von Rhodos. Das Argonautenepos, Bd. 2. Hrsg. und übers. von Reinhold Glei und Stephanie Natzel-Glei. Darmstadt 1996 [3.Jh.v. Chr.].

Badinter, Elisabeth. L'amour en plus. Histoire de l'amour maternel (XVIIe-XXe siècle). Paris 1980.

Balter, Leon. „The Mother as Source of Power. A Psychoanalytic Study of Three Greek Myths“. Psychoanalytic Quarterly 38 (1969): 217-274.

Barthes, Roland. Mythen des Alltags. Übers. von Horst Brühmann. Berlin 2010 [1957].

Bécache, Simone. „Médée“. Revue Française de Psychanalyse 46.4 (1982): 773-793.

Benthien, Claudia und Ortrud Gutjahr. „Interkulturalität und Gender-Spezifik in Tabus. Zur Einleitung“. Tabu. Interkulturalität und Gender. Hrsg. von Claudia Benthien und Ortrud Gutjahr. München 2008: 7-16.

Böschenstein, Renate. „Medea - Der Roman der entflohenen Tochter“. Fathers and Mothers in Literature. Hrsg. von Henk Hillenaar und Walter Schönau. Amsterdam 1994: 7-28.

Böschenstein, Renate. „Medea und die Frage nach der Überzeitlichkeit der Mutterliebe“. Psychoanalyse und die Geschichtlichkeit von Texten. Hrsg. von Johannes Cremerius et al. Würzburg 1995: 127-153.

Cixous Hélène. „Sorties. Out and Out: attacks/ways out/forays“ [1975]. Cixous, Hélène und Catherine Clément. The Newly Born Woman. Übers. von Betsy Wing. Minneapolis 1986: 63-132.

Corbineau-Hoffmann, Angelika. „Medeia“. Mythenrezeption. Die antike Mythologie in Literatur, Musik und Kunst von den Anfängen bis zur Gegenwart. Der Neue Pauly Supplemente, Bd. 5. Hrsg. von Maria Moog-Grünewald. Stuttgart, Weimar 2008: 418-428.

Euripides. „Medeia“ [431 v. Chr.]. Euripides. Sämtliche Tragödien und Fragmente, Bd. 1: Alkestis - Medeia - Hippolytos. Hrsg. von Gustav Adolf Seeck. Übers. von Ernst Buschor. München 1972: 87-182.

Foucault, Michel. Die Ordnung der Dinge. Eine Archäologie der Humanwissenschaften. Übers. von Ulrich Köppen. Frankfurt a. M. 1971 [1966].

Gabriel, Ayala H. „Living with Medea and Thinking after Freud. Greek Drama, Gender, and Concealments". Cultural Anthropology 7 (1992): 346-373.

Grillparzer, Franz. „Das goldene Vließ“ [1822]. Grillparzer, Franz. Werke in sechs Bänden, Bd. 2: Dramen: 1817-1828. Hrsg. von Helmut Bachmaier. Frankfurt a. M. 1986: 205-390.

Haas, Birgit. Das Theater von Dea Loher. Brecht und (k)ein Ende. Bielefeld 2006.

Hidalgo-Xirinachs, Roxana. Die Medea des Euripides. Zur Psychoanalyse der weiblichen Aggression und Autonomie. Gießen 2002.

Horaz [i. e. Quintus Horatius Flaccus]. „De arte poetica liber“ [14 v. Chr.]. Horaz. Sämtliche Werke. Lateinisch - deutsch. Hrsg. von Hans Färber. München 1967: 230-258. 
Hörnigk, Henriette. „,Ich weiß, was du jetzt tun mußt‘. Ein Gespräch mit der Dramatikerin Dea Loher über die Aufführung von Manhattan Medea“. Impuls. Die Zeitschrift des Mecklenburgischen Staatstheaters Schwerin 37 (1999): 6.

Irigaray, Luce. „Körper-an-Körper mit der Mutter“ [1981]. Irigaray, Luce. Genealogie der Geschlechter. Übers. von Xenia Rajewsky. Freiburg i. Br. 1989: 26-46.

Jung, Carl Gustav. Werke. Bd.14.1: Mysterium coniunctionis. Untersuchung über die Trennung und Zusammensetzung der seelischen Gegensätze in der Alchemie. Hrsg. von Lilly Jung-Merker. Ostfildern 1972 [1954].

Kenkel, Konrad. Medea-Dramen. Entmythisierung und Remythisierung. Euripides, Klinger, Grillparzer, Jahnn, Anouilh. Bonn 1979.

Klein, Melanie. Frühstadien des Ödipuskomplexes. Frühe Schriften 1928-1945. Frankfurt a. M. 1991.

Klinger, Friedrich Maximilian. Werke. Historisch-kritische Gesamtausgabe, Bd. 7: Medea in Korinth. Medea auf dem Kaukasos. Aristodymos. Hrsg. von Karl-Heinz Hartmann, Ulrich Profitlich und Michael Schulte. Berlin, Boston 2012 [1786].

Laclau, Ernesto. Emanzipation und Differenz. Übers. von Oliver Marchart. Wien 2002 [1996].

Lesky, Albin. „Medeia“. Paulys Realencyclopädie der classischen Altertumswissenschaft, Bd. 29. Hrsg. von Wilhelm Kroll. München 1931: 30-64.

Lessing, Gotthold Ephraim. „Laokoon: oder über die Grenzen der Malerei und Poesie“ [1766]. Lessing, Gotthold Ephraim. Werke und Briefe, Bd. 5.2: Werke 1766-1769. Hrsg. von Wilfried Barner. Frankfurt a. M. 1990, 9-321.

Leuzinger-Bohleber, Marianne. „Die ,Medea-Phantasie‘. Eine unbewusste Determinante archaischer Aggressionskonflikte bei einigen psychogen sterilen Frauen“. Aggression und seelische Krankheit. Hrsg. von Karin Bell und Kurt Höhfeld. Gießen 1996: 91-121.

Lévi-Strauss, Claude. Strukturale Anthropologie. Übers. von Hans Naumann. Frankfurt a. M. 1967 [1958].

Loher, Dea. „Manhattan Medea“. Loher, Dea. Manhattan Medea. Blaubart - Hoffnung der Frauen. Zwei Stücke. Frankfurt a. M. 1999: 7-62.

Luhmann, Niklas. Liebe als Passion. Zur Codierung von Intimität. Frankfurt a. M. 1982.

Lütkehaus, Ludger. Mythos Medea. Texte von Euripides bis Christa Wolf. Stuttgart 2007.

Luserke-Jaqui, Matthias. Medea. Studien zur Kulturgeschichte der Literatur. Tübingen, Basel 2002.

École de la Cause freudienne - AFG (Hrsg.). La lettre mensuelle 122 (1993).

Mauerer, Gesine. Medeas Erbe. Kindsmord und Mutterideal. Wien 2002.

Müller, Heiner. „Verkommenes Ufer Medeamaterial Landschaft mit Argonauten“ [1983]. Müller, Heiner. Werke, Bd. 5: Die Stücke 3. Hrsg. von Frank Hörnigk. Frankfurt a. M. 2002: 71-84.

Novak, Helga M. Grünheide, Grünheide. Gedichte. 1955-1980. Darmstadt, Neuwied 1983 [1977].

Pape, Wilhelm. „Medeia“. Wörterbuch der griechischen Eigennamen. 2. Aufl., Braunschweig 1884: 910-911.

Palmer, Gesine. „Kein gefallenes Mädchen. Keine Opfermutter. Kein Mutteropfer“. Figurationen 7 (2006): 73-86.

Picasso, Pablo. Variationen über „Las Meninas“von Velazquez eingeleitet durch eine persönliche Betrachtung von Jaime Sabartés. Wien 1960.

Port, Ulrich. „Mythos und Tabu. Über Medeas Mord an ihren Kindern“. Tabu. Interkulturalität und Gender. Hrsg. von Claudia Benthien und Ortrud Gutjahr. München 2008: 101-120.

Pister, David. „Dazwischen: Medea. Zu einer transgressiven Figur(ation) bei Euripides“. Arcadia 48 (2013): 135-149. 
Roos, Esa (Hrsg.). Medea. Myth and Unconscious Fantasy. London 2015.

Schaule, Anton. Tötungshandlungen von Müttern an ihren eigenen Kindern unter besonderer Berücksichtigung des Medea-Komplexes. München 1982.

Schmierer, Britta. Motivation in Medeatragödien der Antike und der Neuzeit. Würzburg 2005.

Seneca [i. e. Lucius Annaeus Seneca]. „Medea“ [um 45 n. Chr.]. Seneca. Sämtliche Tragödien. Lateinisch und Deutsch, Bd.1. Übers. von Theodor Thomann. Zürich, Stuttgart 1961: 239-311.

Simon, Bennett. Tragic Drama and the Family. Psychoanalytic Studies from Aeschylus to Beckett. New Haven u. a. 1988.

Sirola, Riitta. „The Myth of Medea from the Point of View of Psychoanalysis“. Scandinavian Psychoanalytic Review 27 (2004): 94-104.

Saporiti, Sonia. Myth as Symbol. A Psychoanalytic Study in Contemporary German Literature, Part III: „Medea“. Newcastle 2013.

Stephan, Inge. Medea. Multimediale Karriere einer mythologischen Figur. Köln u. a. 2006.

Stern, Edward S. „The Medea Complex. The Mother's Homicidal Wishes to her Child“. Journal of Mental Science 94 (1948): 321-331.

Tyminski, Robert. „The Medea Complex. Myth and Modern Manifestation“. Jung Journal 8.1 (2014): 28-40.

Wiese, Annegret. Mütter, die töten. Psychoanalytische Erkenntnis und forensische Wahrheit. München 1993. 